\title{
Hispanismes
}

Revue de la Société des Hispanistes Français

$17 \mid 2021$

Murs, barrières, obstacles dans les mondes

hispaniques I

\section{Mur de papier, barrière de la langue}

Le cas de la guerre d'imprimés entre la République fédérale centraméricaine et le Honduras britannique (1826-1827)

Muro de papel, barrera de la lengua. El caso de la guerra de impresos entre la República Federal de Centro América y Honduras británico (1826-1827)

A Paper Boarder upon a Language Barrier. The case of the newspaper feud between the Central American Federal Republic and British Honduras

(1826-1827)

\section{Raphaël Roché}

\section{OpenEdition}

\section{Journals}

Édition électronique

URL : https://journals.openedition.org/hispanismes/13470

DOI : 10.4000/hispanismes.13470

ISSN : 2270-0765

Éditeur

Société des Hispanistes Français

Référence électronique

Raphaël Roché, « Mur de papier, barrière de la langue », HispanismeS [En ligne], 17 | 2021, mis en ligne le 01 juin 2021, consulté le 27 janvier 2022. URL : http://journals.openedition.org/hispanismes/13470 ; DOI : https://doi.org/10.4000/hispanismes. 13470

Ce document a été généré automatiquement le 27 janvier 2022.

\section{(c) $(1)$}

Les contenus de cette revue sont mis à disposition selon les termes de la Licence Creative Commons Attribution - Pas d'Utilisation Commerciale - Pas de Modification 4.0 International. 


\section{Mur de papier, barrière de la langue}

Le cas de la guerre d'imprimés entre la République fédérale centraméricaine et le Honduras britannique (1826-1827)

Muro de papel, barrera de la lengua. El caso de la guerra de impresos entre la República Federal de Centro América y Honduras británico (1826-1827)

A Paper Boarder upon a Language Barrier. The case of the newspaper feud between the Central American Federal Republic and British Honduras

(1826-1827)

\section{Raphaël Roché}

\section{Introduction}

Ils s'ront soldats pour la défense

D'la patrie quoi que c'est? C'est la France

La Patrie... ! C'est tuer des Prussiens !

1 Cette citation du poète Gaston Couté illustre bien les deux principaux aspects de la construction d'une nation par le discours, qui consiste d'une part à définir une identité commune, une "communauté imaginée » au sens de Benedict Anderson², et d'autre part à désigner un autre, plus ou moins hostile, qui menace l'existence de la " patrie ». Nous pouvons y voir l'application territoriale de ce que Barth qualifie de "schème général de partition sociale, opérant par position de frontière entre des Nous et des Eux $»^{3}$.

2 Ce travail porte sur l'étude de ces mécanismes, et plus particulièrement du deuxième, à travers un corpus constitué de périodiques publiés en espagnol dans la République fédérale centraméricaine et en anglais dans le Honduras britannique, l'actuel Belize, entre 1826 et 1827. La presse offre un aperçu privilégié du débat public entre les élites pour plusieurs raisons. D'une part, ce sont les traces les plus diffusées et les plus pérennes que les publicistes de l'époque ont laissées, car il s'agit bien, dans tous les cas, d'hommes. Il convient cependant de préciser que la diffusion des périodiques est alors très limitée, puisque leur tirage s'élève à quelques centaines tout au plus ${ }^{4}$. D'autre part, 
la périodicité plus ou moins régulière des publications exige la production d'écrits sur un temps très court, ce qui suppose une spontanéité plus grande que pour un essai ou des mémoires préparés sur le temps long ainsi que, par définition, une prise directe avec l'actualité.

Ainsi, nous nous concentrerons sur les débats qui naissent et se développent entre les publications des deux côtés de la frontière pour essayer d'évaluer leur importance dans la formation d'une identité nationale embryonnaire.

Pour ce faire, il est nécessaire de contextualiser au préalable la situation de l'Amérique centrale et du Honduras britannique de l'époque, d'évoquer les spécificités de leur presse périodique et de mentionner la question de la traduction, indispensable à la compréhension mutuelle des éditeurs de part et d'autre. Par la suite, nous aborderons la place qu'occupe le Belize dans la presse centraméricaine, celle qu'occupe l'Amérique centrale dans la Honduras Gazette puis nous traiterons quelques polémiques qui se sont développées dans cette sphère journalistique avant de nous concentrer sur la question de l'esclavage et de sa relation avec la morale religieuse, qui cristallise toutes les tensions entre l'Amérique centrale et le Honduras britannique.

\section{La République fédérale centraméricaine et le Honduras britannique en 1826}

Nous allons évoquer tour à tour la situation dans ces deux territoires qui est très différente culturellement et institutionnellement.

D’une part, la République fédérale centraméricaine - República Federal de Centro América - s'est constituée à l'issue du long processus indépendantiste de l'ancienne capitainerie générale de Guatemala. Celle-ci se matérialise d'abord par la déclaration d'indépendance vis-à-vis de l'Espagne du 15 septembre 1821, obtenue sans combats contre les autorités coloniales, mais sous la double pression de l'homme fort du Mexique, Agustín de Iturbide, et d'une partie des habitants de la ville de Guatemala. Le préambule et le premier article de la déclaration d'indépendance en témoignent: « $1^{\circ}$ Que siendo la independencia del gobierno español la voluntad general del pueblo de Guatemala [...], el Sr. jefe político la mande publicar para prevenir las consecuencias que serían temibles en el caso de que la proclamase de hecho el mismo pueblo ${ }^{5}$. De fait, cette indépendance ne dure que quelques mois, puisqu'une assemblée provisoire vote dans des conditions difficiles l'union - ou l'annexion - à l'empire mexicain en janvier 1822, union qui est annulée à la suite de la chute de l'Empire en juillet $1823^{6}$. À ce moment, une Assemblée nationale constituante se réunit et opte pour la solution fédérale avec la proclamation de la Constitution le 22 novembre 1824 . Le système se met alors progressivement en place et un premier président fédéral, des chefs d'États fédérés ainsi que des parlements commencent à siéger. Dans un tel contexte, l'objectif de ces politiciens est simplement de faire en sorte que cette nouvelle entité survive, ce qui n'est pas chose aisée compte tenu de nombreuses difficultés internes et externes. D'un côté, l'Amérique centrale craint des velléités de reconquête de la part des deux puissances qui l'ont dominée au cours des années précédentes, l'Espagne et le Mexique $^{7}$. De l'autre côté, des tensions existent entre différents États fédérés, principalement entre le Guatemala - dont la capitale est le siège des autorités politiques en plus d'être l'État le plus peuplé et le plus prospère - et le reste des 
membres de la fédération. Ce sont ces difficultés internes qui sont sans doute les plus préoccupantes, puisqu'elles mèneront à une guerre civile qui s'étend de la toute fin de l'année 1826 à $1829^{8}$. Au moment où elle prend forme, la République fédérale centraméricaine compte entre 1 et 2 millions d'habitants, dont une majorité d'indigènes, pour la plupart mayas, peu impliqués dans la vie publique de cet État criollo ${ }^{9}$.

D'autre part, le Honduras britannique est une colonie de la Couronne, administrée par un gouverneur. Cet établissement s'est construit à partir d'un repaire de pirates anglais au début $\mathrm{du}$ XVII ${ }^{\mathrm{e}}$ siècle sur la côte caribéenne de l'Amérique centrale, délaissée par la colonisation espagnole. L'établissement va se développer de manière significative à partir de 1670, qui est une date importante pour deux raisons. En premier lieu, il s'agit de l'année du traité de Madrid qui acte la cession à la Grande Bretagne des territoires espagnols occupés de facto par Londres, sans pour autant préciser quels sont les territoires en question, ce qui ouvre la voie à de nombreux conflits. En second lieu, l'année 1670 marque l'essor de l'exploitation des bois précieux de la région, l'acajou et le campêche. La colonie anglaise puis britannique est en lien étroit avec la Jamaïque, qui va constituer sa source principale de peuplement ${ }^{10}$. À l'époque que nous étudions, la population du Honduras britannique est considérablement moins nombreuse que la centraméricaine puisqu'elle ne compte qu'environ 5000 habitants, dont une majorité d'esclaves afro-descendants ${ }^{11}$. C'est en cela un établissement colonial démographiquement bien plus proche de la Caraïbe britannique que de l'Amérique centrale.

8 Au sujet de la relation entre les deux territoires, il est important de préciser que les autorités espagnoles, puis centraméricaines et guatémaltèques ne reconnaissent pas l'indépendance du Belize, ce qui est un point fondamental qui s'explique par l'ambiguïté des termes du traité de Madrid évoqué plus haut.

\section{Le corpus : la presse périodique}

9 L'Amérique centrale et le Honduras britannique sont, également sur ce point, à des stades de développement très distincts concernant la presse périodique. Guatemala est la quatrième ville hispano-américaine à disposer d'une imprimerie en 1660 et San Salvador se joint à elle en $1824^{12}$. La presse périodique s'est développée dès la fin du XVIII ${ }^{\mathrm{e}}$ siècle avec une première publication - la Gazeta de Guatemala - que l'on pourrait qualifier d'ilustrada, caractéristique de l'Amérique coloniale, dans laquelle domine une tonalité scientifique et didactique (voire encyclopédique) bien plus que le contenu d'actualité au sens propre $^{13}$. Le journalisme connaît ensuite deux phases de développement de 1820 à 1822 d'abord, quand deux périodiques paraissent en même temps pour la première fois ${ }^{14}$ et après 1824 , où paraissent cette fois en même temps cinq publications distinctes qui sont toutes de tonalité politique. La première est la gazette officielle, la Gaceta del Gobierno Supremo de Guatemala, la deuxième est salvadorienne, le Semanario político mercantil de San Salvador, et les trois autres défendent des options politiques différentes, puisque $E l$ Liberal est libéral radical, $E l$ Indicador modéré et que le Redactor General est l'œuvre personnelle d'un politicien de premier plan cherchant à se placer au-dessus des partis, José Cecilio del Valle ${ }^{15}$. Parmi tous ces périodiques, nous étudierons ici surtout la publication modérée El Indicador, 
car il s'agit de la plus pérenne - 156 numéros en 1824 et 1827 - et de celle qui couvre le plus la période de publication de son homologue bélizien ${ }^{16}$.

Du côté du Honduras britannique, la faible population et l'importance stratégique négligeable de l'établissement à l'échelle de l'Empire colonial britannique expliquent que l'imprimerie et la presse ne fassent pas leur apparition avant l'année 1826, date de parution de la Honduras Gazette and Commercial Advertiser. C'est l'œuvre d'un commerçant probablement d'origine écossaise, James Cruickshank, dont les sautes d'humeur répétées vis-à-vis des autorités coloniales lui vaudront d'être exproprié un an et demi plus tard, après avoir édité 88 numéros. Cependant, cette première publication est d'une importance significative eu égard à son contenu original.

11 Mis en regard, El Indicador et la Honduras Gazette présentent des différences significatives, puisque le périodique centraméricain contient en majorité des articles à dimension politique et liés à l'actualité au sens large, alors que la publication bélizienne offre un visage nettement plus mercantile, la première page étant systématiquement constituée d'annonces commerciales concernant la vie du port. Les deux périodiques s'échangent des articles polémiques, mais la plume de la Honduras Gazette est bien plus acérée et provoque les attaques les plus fortes, ce qui est sans doute à mettre en relation avec l'influence de la tradition journalistique britannique et avec la personnalité de son éditeur et auteur principal.

\section{Fabriquer et traduire la presse}

Si l'on se place du point de vue centraméricain, le Honduras britannique constitue l'unique puissance frontalière culturellement et linguistiquement différente, puisque les autres voisins de la République fédérale sont le Mexique et la Colombie. Pour le Belize, l'Amérique centrale est le seul État limitrophe avec qui des relations importantes sont entretenues. D'un côté comme de l'autre, la langue, la religion et le système politique constituent autant de manières de se différencier du voisin. Cependant, la nature du corpus étudié met la question de la langue au premier plan, du fait des interactions entre éditeurs anglophones et hispanophones.

Il est nécessaire de donner au préalable quelques éléments sur le contenu des périodiques de l'époque. L'importance du contenu original, c'est-à-dire d'articles écrits expressément pour la publication périodique, peut varier mais il ne constitue habituellement qu'une partie minoritaire de l'ensemble d'un numéro. Avant l'apparition des agences de presse, liée au développement du télégraphe, les éditeurs de la presse périodique trouvent leur principale source d'information en lisant d'autres périodiques et en reprenant certains de leurs articles, toujours sourcés par le titre de la publication, son numéro ou sa date de parution. De manière plus occasionnelle, les éditeurs peuvent également avoir recours à la reprise de fragments d'œuvres publiées, souvent s'ils ne disposent pas de nouveaux journaux à consulter ${ }^{17}$. Cela est très clairement observable pour les derniers numéros de la Honduras Gazette que nous incluons dans cette analyse. Au cours de sa première année, la publication est hebdomadaire et s'alimente de nouveaux périodiques reçus grâce aux malles qui sont régulièrement chargées dans les bateaux mouillant dans le port de Belize, les liaisons terrestres avec le reste du continent étaient très difficiles. Ces malles contiennent surtout des journaux jamaïcains, britanniques et états-uniens, mais aussi latinoaméricains. Après le premier anniversaire de la publication, l'éditeur décide de passer 
d'un à deux numéros hebdomadaires, ce qui crée une baisse significative de la qualité du journal. Ainsi, ne disposant plus de nouvelles fraîches, il insère du contenu ayant de moins en moins de rapport avec l'actualité, l'exemple le plus éloquent étant certainement un article sur la « vraie gloire » - «Definition of True Glory » - (numéro 82, 10/10/1827) issu d'un écrit de l'abbé de Raynal, mort trente ans plus tôt. Cette pénurie de matériel original est moins notable dans le cas centraméricain, dont le territoire est alors plus en contact avec l'étranger grâce à des liaisons routières certes déficientes, mais plus praticables, avec le Mexique et à plusieurs ports se situant sur les côtes caraibe et pacifique.

Cet exemple d'article repris de Raynal dans le journal en anglais d'un établissement colonial de quelques milliers d'habitants pose la question de la langue et de la traduction d'articles étrangers vers l'espagnol ou l'anglais. Or, il est intéressant d'observer de bonnes compétences linguistiques et traductologiques en Amérique centrale comme au Honduras britannique, puisque El Indicador, tout autant que la Honduras Gazette, reprend des articles de l'autre langue en les traduisant de manière tout à fait exhaustive et précise, sans omission, manipulation ni contournement de difficultés.

On observe même la publication de certaines annonces à caractère commercial en espagnol - sans qu'aucune faute n'apparaisse - dans la Honduras Gazette, qui ciblent les Centraméricains venus acheter des produits britanniques. Nous pouvons raisonnablement penser que ces annonces sont rédigées à l'aide d'un ouvrier hispanophone, détail que révèle l'éditeur de la gazette plus tard à l'occasion de sa disparition. Cruickshank offre une récompense à qui pourra lui donner des informations à son sujet (numéro 77, 22/09/1827). Précisons que cet article fait partie des rares indications que les périodiques dévoilent au sujet de leur propre élaboration.

Nous allons à présent étudier la place qu'occupe le Honduras britannique dans la presse centraméricaine, puis celle de l'Amérique centrale dans la Honduras Gazette.

\section{Quasi-invisibilité du Belize dans la presse centraméricaine}

17 Aucune référence à l'établissement britannique ne peut être trouvée dans El Indicador avant l'apparition du premier numéro de la Honduras Gazette. Une recherche étendue à un échantillon de l'ensemble de la presse centraméricaine de 1824 à $1826^{18}$ confirme ce que nous pouvons considérer comme une quasi-invisibilité. Seule la publication officielle mentionne la colonie dans les comptes-rendus de l'activité du port d'Omoa situé au Honduras, non loin de la ville de Belize - et dans un article évoquant la visite d'un émissaire en provenance du territoire auprès du gouvernement centraméricain en 1825 (Gaceta del Gobierno Supremo de Guatemala, numéro 39, 27/03/1825).

Cette absence totale du Belize dans El Indicador avant 1826 et presque totale dans le reste de la presse centraméricaine n'est pas très étonnante compte tenu du contexte. En effet, puisque la République fédérale centraméricaine établit de nouvelles institutions, ce sont les questions politiques internes qui occupent les éditeurs, d'autant plus qu'ils sont eux-mêmes politiciens. Du point de vue de l'actualité internationale, l'attention des Centraméricains est accaparée par d'autres sujets, particulièrement par les relations compliquées avec le Mexique, ancienne puissance impériale avec qui les 
différends territoriaux du Chiapas et du Soconusco ne sont toujours pas résolus. De plus, une partie de la presse mexicaine s'emploie à tourner en ridicule l'existence d'un État centraméricain et à affirmer que seul un rattachement au Mexique est viable à terme ${ }^{19}$. Un deuxième élément peut expliquer la place dérisoire du Belize dans la presse centraméricaine, à savoir le relatif abandon du Petén, région frontalière du territoire britannique, qui n'est alors presque pas colonisée par les criollos. Les contacts sont très occasionnels et n'ont que très peu de répercussions pour les éditeurs de périodiques qui habitent la capitale. À cet effet, nous pouvons mentionner un article qui énumère les conflits existant aux frontières de l'État du Guatemala (El Indicador, numéro 47, 12/09/1825). L'auteur y évoque deux différends, un premier interne à la République fédérale avec El Salvador au sujet de la région de Sonsonate, et un deuxième, international, avec le Mexique autour de la région frontalière du Soconusco. Le Honduras britannique, dont le territoire est pourtant censé être revendiqué par la République fédérale, n'y est pas mentionné.

e place considérable dans l'ensemble de la presse centraméricaine de l'époque pour plusieurs raisons. D'abord, il s'agit du modèle admiré par tous les pays nouvellement indépendants du fait de son expansion économique, de son pouvoir financier, maritime, diplomatique, de sa tradition démocratique - appliquée à la Grande-Bretagne bien plus qu'à son empire - et de son prestige intellectuel. En effet, elle constitue le berceau du libéralisme et d'une autre doctrine philosophique en vogue chez les politiciens latino-américains de cette génération, l'utilitarisme de Jeremy Bentham ${ }^{20}$.

De plus, l'un des premiers objectifs des États nouvellement indépendants consiste à être intégré au "concert des nations $»^{21}$, ce qui passe formellement par l'obtention de la reconnaissance de leur gouvernement par d'autres États, si possibles influents. Ainsi, dans le contexte de l'époque, ce sont surtout les grandes puissances libérales qui sont visées, au premier rang desquelles figurent le Royaume-Uni, les États-Unis et dans une moindre mesure les Pays-Bas, étant entendu que tous les États hispano-américains ont intérêt à se reconnaître mutuellement. Les autres puissances continentales (Espagne, France, Autriche, Prusse et Russie) sont alors des monarchies conservatrices, voire réactionnaires, qui se montrent solidaires de l'Espagne, résolue à ne pas reconnaître l'indépendance de ses anciennes colonies.

21 Cette quasi-inexistence du Honduras britannique dans la presse centraméricaine va cesser avec la parution de la Honduras Gazette, qui va consacrer une partie importante de son contenu à la République fédérale.

\section{Omniprésence de l'Amérique centrale dans la Honduras Gazette}

L'État centraméricain, mentionné dès le premier numéro de la Honduras Gazette (le $1^{\mathrm{er}}$ juillet 1826), apparaît dans la presque totalité des 88 numéros étudiés. On observe clairement deux étapes dans le traitement de la République fédérale par l'éditeur de la Gazette, qui sont liées au déclenchement de la guerre civile dans ce pays. Avant le début des actions militaires, Cruickshank attaque frontalement l'Amérique centrale, remet en cause son existence et sa viabilité comme État indépendant. Par ailleurs, il est intéressant de noter que la publication comprend également des articles évoquant des sujets hors-actualité pour renforcer l'idée d'une hostilité entre Amérique centrale et 
Belize, à l'instar d'une histoire des affrontements militaires entre la Grande-Bretagne et l'Espagne pour le contrôle du territoire bélizien (numéros 12, 16/09/1826, et suivants).

Un point d'inflexion est atteint au début de l'année 1827, quand il devient clair aux yeux des observateurs que l'opposition politique à l'origine de la révolte armée n'a pas pu être définitivement abattue par le pouvoir et qu'un conflit au long cours s'impose (le dernier article très critique de la République fédérale dans son ensemble figure au numéro 31 en date du 27 janvier). Une fois que la guerre est véritablement déclenchée entre le gouvernement central, modéré, soutenu surtout par le Guatemala et un parti libéral plus radical défendu par le Honduras, le Nicaragua et El Salvador, le ton agressif de la Gazette disparaît complètement. La couverture de l'Amérique centrale se fait alors beaucoup plus factuelle et concernée par les conséquences que le conflit interne chez ce grand voisin peut avoir sur l'établissement bélizien. Ainsi, la situation militaire est largement abordée, et le contenu plus politique consiste à appuyer le président en place, Manuel José Arce, qualifié d'homme sérieux, car respectant l'intégrité de la colonie britannique, contrairement à ses rebelles radicaux. Dans cette deuxième phase, le ton agressif n'est plus destiné qu'à ces derniers, ce qui marque un engagement total de la Honduras Gazette pour le gouvernement de l'État qu'elle critiquait encore quelques mois auparavant.

\section{Guerres de papier}

Ce sont donc les échanges précédant la guerre civile qui vont nous intéresser plus particulièrement ici. Il convient de noter que des écrits très offensifs au sujet de l'Amérique paraissent dès les premiers numéros de la Honduras Gazette. Dans le numéro 3 (15/07/1826), un long article attaque l'État centraméricain sur tous les fronts, avec trois arguments forts.

Le premier, historique, tourne le processus indépendantiste centraméricain en ridicule pour donner l'image d'un pays faible. Ainsi, l'éditeur passe en revue l'indépendance provisoire qui s'est transformée en annexion par le Mexique, la petite taille de l'armée nationale dont l'autorité a chancelé à la suite d'une tentative de coup d'État en 1823.

Le deuxième argument tient à la population ainsi qu'au développement économique et général du pays. Il décrit une Amérique centrale dépeuplée qui ne compterait que trois «vraies » ou « bonnes » villes («three good towns »), et ajoute que « les neuf dixièmes [du territoire] sont incultes [...] remplis de noms de villes et de villages grandiloquents dont seul le nom existe, ou qui ne sont que des regroupements de huttes qui feraient passer les cabanes de la plus pauvre paysannerie irlandaise pour des palais en comparaison $»^{22}$. Cette citation nous permet de mentionner le style très incisif de l'éditeur Cruickshank dans ces articles, qui emploie un grand nombre de procédés pour appuyer son argumentation, comme des comparaisons hyperboliques, des phrases exclamatives, ainsi que des mises en relief typographiques (italiques ou majuscules).

Le dernier argument, qui n'est pas le moindre, s'attaque directement au sérieux des institutions centraméricaines et à leur viabilité. Le Congrès centraméricain est ainsi qualifié d'hostile, car il souhaite débaucher les travailleurs béliziens, le gouvernement est lui aussi disqualifié, puisqu'il est décrit comme "putatif» («would-be government »), tout comme l'État dans son ensemble, qui ne tiendrait debout que grâce à un prêt accordé par une banque londonienne, avec le soutien de la Couronne. L'idée 
sous-jacente est que Londres ne soupçonne pas la situation réelle en Amérique centrale, sans quoi aucune aide ne lui serait apportée. Cela est résumé à la fin de l'article par une affirmation péremptoire : « nous avons donné une image véritable du Guatemala. Nous connaissons son imbécillité, nous savons qu'il manque de tout ce qui est essentiel pour faire une nation et que sa chute est proche - si la Grande-Bretagne ne le préserve pas $»^{23}$.

Les réactions que cet article va susciter sont considérables du côté centraméricain. Sa première conséquence est d'accorder au territoire bélizien une place importante dans la presse centraméricaine, bien que nous ayons vu qu'elle était auparavant réduite à la portion congrue. Ainsi, pour El Indicador, le Honduras britannique fait l'objet de six articles entre le numéro 94 (numéro 24/08/1826) et la disparition de la publication (numéro 156, 06/11/1827) alors qu'il est totalement absent des 93 premiers numéros. Ce sont donc les offensives de la Honduras Gazette qui viennent mettre l'établissement colonial sur la carte mentale des éditeurs centraméricains. Le premier article qui vient répondre au numéro 3 de la Gazette (El Indicador, numéro 94, 24/08/1826) constitue une réplique importante tant au niveau de sa longueur que de sa teneur. En effet, il s'agit d'un double numéro de huit pages au lieu de quatre, fait exceptionnel qui n'a lieu que trois fois pendant la publication du périodique. De plus, ce double numéro est construit autour de l'article venant répondre à l'attaque de la Honduras Gazette, intitulé «Impresos de fuera de la República ». Il s'agit également du plus long de toute la publication avec près de 5,5 pages. Le dispositif n'est pas habituel pour l'observateur contemporain étant donné que l'article d'origine est - bien et intégralement - traduit, alors qu'il aurait été envisageable de supprimer certains passages ou de forcer le trait pour caricaturer l'adversaire. Nous pouvons remarquer quelques notes de traduction rendant visible le travail de l'éditeur lorsqu'il veut souligner une difficulté d'ordre linguistique, dans une volonté d'être le plus transparent possible, puisque le lecteur polyglotte peut retrouver ces difficultés dans le texte original.

Dès le début de l'article, les auteurs évoquent un écrit «sumamente injurioso para esta República ». La réponse à cet article de la part des Centraméricains se fait par notes de bas de page, qui est un dispositif fréquent lors des guerres de papier, ces polémiques qui naissent entre éditeurs de périodiques. Le ton des éditeurs de El Indicador est subtil, car leur objectif est complexe. Leur intention semble être de répondre coup pour coup aux arguments raillant la non-viabilité et le retard de la République fédérale, sans attaquer de front la Grande-Bretagne en tant que telle. Il ne faut pas perdre de vue l'objectif des politiciens centraméricains, à savoir l'obtention de la reconnaissance de leur État, qui garantirait leur survie sur la scène internationale. Dans ce contexte, une attaque du bien-fondé de la présence britannique au Belize serait plus que malvenue. De fait, les éditeurs estiment que l'objectif initial de la Honduras Gazette est de nuire à l'image de l'Amérique centrale auprès du gouvernement britannique. Une lutte d'influence semble donc initiée pour gagner le soutien de Londres.

El Indicador prend soin de répondre à toutes les critiques de Cruickshank de manière assez honnête, sans nier les difficultés réelles auxquelles la République fédérale centraméricaine est confrontée. Ainsi, si nous reprenons les trois arguments principaux synthétisés plus haut, les éditeurs centraméricains font valoir que le processus indépendantiste a certes été complexe et instable, mais que ce genre d'incidents est caractéristique des pays nouvellement indépendants, et plus généralement de ceux traversant des épisodes révolutionnaires. La description d'une 
terre dépeuplée, inculte est balayée par les Centraméricains qui considèrent que cette critique - déjà formulée par des périodiques mexicains - est en décalage total avec la réalité et ne résisterait pas à un court séjour dans le pays. Enfin, l'idée que les institutions étatiques seraient à la fois non viables et condamnées à l'échec sans le soutien britannique reçoit une réponse plus nuancée. Les éditeurs admettent la fragilité du nouveau pays, surtout face à des voisins plus puissants comme la Colombie et surtout le Mexique. Ils s'accordent à dire que la reconnaissance de la Fédération par la Grande-Bretagne est effectivement la seule solution pour sa survie à long terme. En ce qui concerne la dépendance vis-à-vis de la dette britannique, les éditeurs la remettent en cause, dans la mesure où l'argent n'a été que très partiellement versé.

31 La réponse à la critique finale est assez habile, les éditeurs soulignant le décalage entre le peu d'importance que l'Amérique centrale revêt selon Cruickshank et son long développement qui tente laborieusement de le démontrer. Les derniers paragraphes attaquent particulièrement l'éditeur de la Honduras Gazette et le gouvernement bélizien - qualifié de "gobierno subalterno »-, en rappelant sa faible puissance au regard de l'Amérique centrale, pourtant rabaissée. Cependant, ils veillent bien à le séparer du gouvernement britannique qui n'est cité qu'en termes élogieux: "concluimos suplicando a los señores editores de la Gaceta de Walis ${ }^{24}$, que imitando la conducta de su nación y de su alto gobierno, respeten a un país que es más acreedor que otro alguno a las consideraciones de un establecimiento que le debe todo su ser $"$.

Ces répliques sont intéressantes, car elles permettent à la sphère journalistique centraméricaine, prompte à se diviser sur des questions partisanes, d'appeler à l'unité nationale entre les différents journaux de la République. Il convient ici de rappeler une évidence, à savoir que la désignation d'un ennemi ou d'une menace externe agit comme un ferment très efficace pour l'union d'une communautée ${ }^{25}$.

Ainsi, ces réponses à des articles remettant en cause la viabilité de la République de la Honduras Gazette ou d'autres grands journaux étrangers comme $\mathrm{El} \mathrm{Sol,} \mathrm{périodique}$ mexicain de référence à l'époque, sont souvent placées sous le titre " Honor nacional ». De plus, ces articles donnent lieu à la constitution d'un argumentaire de plus en plus rodé, ce que l'on observe à travers les renvois des éditeurs vers les numéros précédents, ce qui est le cas lorsque les éditeurs répondent aux attaques ciblant la faible population de la République fédérale et la faible exploitation des ressources. Dans de telles circonstances, ces journalistes renvoient leurs lecteurs aux réponses apportées par le passé à des attaques similaires provenant de périodiques mexicains.

Les guerres d'imprimés occasionnées dans ce cas par des articles incisifs de la Honduras Gazette et saisies au bond par les éditeurs centraméricains ont néanmoins pour base une question centrale qui concentre les crispations, à savoir le statut de l'esclavage au Honduras britannique.

\section{L'esclavage et la morale comme principaux sujets d'opposition}

Il convient de revenir sur un point évoqué plus haut parmi les nombreuses critiques adressées par Cruickshank à l'Amérique centrale, le supposé "débauchage » des travailleurs du Belize par les autorités centraméricaines, qui sont en réalité des esclaves vitaux pour l'économie de la colonie. En effet, cette critique est directement 
liée à l'abolition de l'esclavage par la République fédérale centraméricaine, qui est l'une des mesures symboliques prises par les institutions le 17 avril 1824 et qui sera réaffirmée dans la constitution fédérale. Notons que du point de vue centraméricain, l'abolition de l'esclavage constitue un geste généreux à peu de frais, dans la mesure où il n'y a que peu d'esclaves au sens juridique du terme - environ 500 dans tout le pays et que l'économie locale s'appuie sur le travail de la population indigène, ce qui n'est pas considéré comme de l'esclavage ${ }^{26}$. La question de l'appel à la désertion, dénoncé par Cruickshank et les autorités béliziennes, est liée à la libération de fait des esclaves étrangers qui trouveraient asile sur le territoire centraméricain.

La Honduras Gazette se sert de cette mesure pour dénoncer un idéalisme libéral radical, ce qui représente par ailleurs un argument souvent utilisé par les conservateurs ou les modérés de l'époque contre les libéraux radicaux dans le contexte hispano-américain. Ainsi, la publication coloniale tourne en dérision un des meneurs du parti libéral, qu'elle décrit comme un philanthrope tellement empli de bonté qu'il souhaite légiférer pour le monde entier ${ }^{27}$ (numéro 23, 02/12/1826). Par conséquent, il est clair que l'attitude agressive de l'éditeur bélizien vise plus particulièrement le mouvement radical, qui n'est pas au pouvoir en début 1826, mais qui sera fortement représenté par les rebelles durant la guerre civile centraméricaine.

Cette vision duale de la classe politique centraméricaine est exposée pour la première fois dans le numéro 8 de la publication bélizienne (19/08/1826). Bien que la guerre civile n'ait pas encore débuté, le correspondant de la Gazette en Amérique centrale fait état de graves tensions qui émergent entre le pouvoir législatif radical et l'exécutif, modéré. Cependant, l'auteur ne craint pas de violence, car il considère que le peuple centraméricain est d'un caractère soumis et indifférent aux affaires publiques. Cet article suscite donc notre intérêt surtout parce qu'il inclut la première référence positive à une partie des acteurs politiques centraméricains, en évoquant l'existence d'un parti "véritablement patriotique ", celui qui désire que justice soit rendue aux habitants de l'établissement colonial. L'auteur explicite davantage son propos en se référant directement au retour des esclaves enfuis et affranchis en Amérique centrale vers leurs propriétaires « légitimes ${ }^{28}$.

Un deuxième article publié un peu plus tard dans la Gazette (numéro 15, 07/10/1826) va plus loin dans cette opposition qui est établie entre l'exécutif, et particulièrement le président de la République fédérale, décrit comme responsable et le législatif, formé de députés idéologues, ignorant toute notion de bien public ${ }^{29}$. Il est intéressant de souligner que cet article vient répondre à un numéro du périodique centraméricain $\mathrm{El}$ Liberal (en date du 20/08/1826) ${ }^{30}$, qui avait lui-même été écrit en réaction à l'article du numéro 8 de la Gazette cité plus haut. Il n'est pas étonnant que les éditeurs béliziens aient retenu cette publication centraméricaine comme cible, puisqu'il s'agit de l'organe du parti qu'il dénonce au premier chef. Ainsi, le fait qu'il s'agisse d'un débat engagé contre une publication considérée comme hostile aiguise certainement le contenu de la critique qui part de la controverse sur l'esclavage et l'étend à d'autres points.

De fait, après avoir dissocié le président du parlement qu'il associe aux éditeurs de $E l$ Liberal, Cruickshank dénonce une fois de plus la grandiloquence d'un parti qui se targue d'avoir aboli rapidement l'esclavage là où cette question est débattue depuis des décennies en Europe. C'est à ce moment que l'argumentation de l'éditeur de la Honduras Gazette prend une tournure intéressante, lorsqu'il dénonce le décalage entre cette politique dénoncée plus haut et le catholicisme affiché de la République fédérale, qui va 
de pair avec le poids considérable du clergé dans la vie quotidienne du pays et de sa capitale en particulier. À cet effet, précisons que l'éditeur britannique a raison au sujet du poids de la religion catholique dans l'Amérique centrale de l'époque, ce que nous pouvons illustrer avec quelques exemples. Le mois qui suit l'annulation de l'union au Mexique, en juillet 1823, la devise « Dios, Unión, Libertad » est adoptée par l'Assemblée nationale constituante majoritairement radicale. Un peu plus d'un an plus tard, la Constitution est proclamée "en nombre del ser supremo, autor de las sociedades y legislador del universo » et son article 11 déclare que la religion d'État est la catholique et que l'exercice public des autres cultes n'est pas permis ${ }^{31}$. Enfin, une publication guatémaltèque satirique de la même période ironise sur l'impossibilité de marcher plus de cent mètres sans rencontrer un ecclésiastique :

La abundancia del pasto espiritual con que nos convida por todas partes, que [no] anda U[sted. una manzana entera, sin encontrarse con un Monasterio, o alguna Iglesia filial, ni avanza un paso en la carrera, sin dejar de tropezarse con un monje, un monigote, o con una beata decrépita, de que por fortuna estamos tan abastecidos para honra y gloria de Dios $^{32}$.

Cette situation est exploitée par l'éditeur de la Gazette qui voit dans l'Amérique centrale un État religieux qui ne prend même pas la peine de respecter le $8^{\mathrm{e}}$ commandement : tu ne voleras point. La politique d'asile et d'affranchissement des esclaves étrangers est donc considérée comme du vol pur et simple. La critique est poussée encore plus loin lorsque les éditeurs ajoutent que les politiciens centraméricains, étant incapables de mettre sur pied une force de police digne de ce nom, laissent régner un niveau de violence tel que le poignard est devenu un accessoire aussi indispensable qu'une cape dans la capitale. Ce laxisme rend ainsi les députés centraméricains coupables de ne pas respecter un autre commandement, le $6^{\mathrm{e}}$ : tu ne tueras point. Cet argument vaut un soutien étrange au président de la République fédérale Manuel José Arce de la part des éditeurs béliziens, qui considèrent que ce dernier rétablirait l'ordre s'il en avait les moyens. Mais les éditeurs béliziens ne vont pas s'arrêter à ces critiques. Ils vont jusqu'à affirmer que la République fédérale centraméricaine est un État fantoche manipulé par l'Espagne, une " colonie secrète $»^{33}$, car elle ne fait pas la guerre à l'Espagne à l'inverse de ses deux grands voisins, le Mexique et la Colombie, et que les deux seuls navires détenus par la Fédération relieraient l'Amérique centrale à La Havane en hissant des pavillons espagnols. Observons que cette accusation, basée sur de bien maigres éléments, entre directement en contradiction avec le reste de l'article et la ligne éditoriale de la Honduras Gazette, puisque le libéralisme dogmatique que la publication prête aux députés radicaux centraméricains a peu de choses en commun avec la dernière phase, absolutiste, du règne de Ferdinand VII. Au-delà de ce fait, l'association de l'Amérique centrale à l'Espagne n'est pas anodine, dans la mesure où les arguments employés, comme la critique du catholicisme ou la dénonciation d'une volonté hégémonique de la part de Madrid, sont caractéristiques de la légende noire diffusée en partie par la Grande-Bretagne au cours des siècles précédents ${ }^{34}$. D'autres renvois au passé colonial espagnol sont par ailleurs présents dans la publication bélizienne, à l'image d'un long article filé sur plusieurs numéros, cité plus haut, qui évoque les guerres du siècle antérieur entre Espagne et Royaume-Uni pour le contrôle du Honduras britannique (« Historical Sketches of Honduras », numéros 12, 16/09/1826, et suivants).

41 Les éditeurs d'El Indicador (numéro 107, 20/11/1826) cherchent encore une fois à répondre à ces critiques portant sur l'esclavage de manière nuancée, sans suivre le ton 
acerbe et l'analyse hyperbolique de la Honduras Gazette. De plus, ils saisissent l'occasion qui leur est offerte de défendre les positions de leurs alliés modérés et du président de la République fédérale, alors que les Béliziens les ménagent. Ainsi, les Centraméricains insistent sur la réponse favorable du parti modéré à la demande officielle de la part du Belize pour le retour des esclaves qui se sont enfuis. Cette demande a été traitée par le Parlement, mais bloquée par les libéraux, et $E l$ Indicador - quoiqu'en désaccord avec cette résolution - fait valoir que la Grande-Bretagne doit respecter la vie démocratique des pays étrangers, comme ces derniers doivent respecter les décisions britanniques.

\section{Conclusion}

Nos recherches ont mis en évidence l'importance de l'imprimé dans la construction des projets nationaux de l'époque de l'indépendance hispano-américaine. En effet, dans le cas de la presse centraméricaine, ce sont les attaques de la Honduras Gazette qui ont donné au Belize une place dans la sphère publique qui, jusqu'alors, n'existait presque pas. En cela, un mur de papier se crée au travers de la barrière de la langue, par la traduction d'articles écrits en anglais vers l'espagnol et vice-versa. En outre, le discours alimenté par ce périodique permet un travail de défense rhétorique de la part des éditeurs centraméricains, qui militent pour l'union des élites par le truchement des différentes publications de l'époque. Nous pouvons d'ailleurs considérer que ces tentatives, dérisoires, n'ont pas été couronnées de succès, comme le suggère l'éclatement de la guerre civile qui a lieu à la fin de la période étudiée. Empressonsnous de préciser cependant que si les tensions entre ces éditeurs étaient réelles, elles ne peuvent être considérées que comme une cause indirecte de la guerre civile, qui s'explique par bien d'autres facteurs à l'instar des frictions entre les autorités fédérales et fédérées. Néanmoins, la constitution de discours publics de la part des élites est révélatrice de la volonté de forger une identité nationale centraméricaine, étant entendu que "la véritable naissance d'une nation, c'est le moment où une poignée d'individus déclare qu'elle existe et entreprend de le prouver $\aleph^{35}$.

Enfin, nous observons que les périodiques se lancent dans une guerre pour tenter d'influencer les puissants. C'est le cas à la période étudiée lorsque la Honduras Gazette et El Indicador semblent se battre pour obtenir la reconnaissance - ou la nonreconnaissance - de l'indépendance centraméricaine par le gouvernement britannique. Les éditeurs d'un périodique jouent un rôle bien limité, ne pouvant que diffuser un discours symbolique auprès d'une audience très réduite. Cependant, il semble que les journalistes eux-mêmes se rendent compte à l'époque de cette relative impuissance, comme le laisse entendre le journaliste centraméricain Antonio José de Irisarri dans le premier numéro d'une publication qui paraît quelques années après la guerre civile : «Mi periódico servirá de lo que sirven en los buques mercantes los cañones de palo que hacen el artificio de la artillería y dan respeto al buque, haciendo que el que los ve de lejos se engañe con su apariencia $»^{36}$. 


\section{BIBLIOGRAPHIE}

«Acta de independencia del antiguo reino de Guatemala », in José Cecilio DEL VALLE, Obra escogida, Caracas, Biblioteca Ayacucho, 1982, p. 3-6.

Benedict ANDERSON, L'Imaginaire national : réflexions sur l'origine et l'essor du nationalisme, traduit par Pierre-Emmanuel Dauzat, Paris, La Découverte, 1996.

ASAMBlea nacional CONSTITUYente, Constitución de la República federal de Centro América dada por la Asamblea Nacional Constituyente en 22 de noviembre de 1824, Facismilé de l'édition de 1824. Guatemala, Imp. Marroquín Hnas., 1956.

John BRownING, «Desarrollo del periodismo », in Historia General de Guatemala. T. 4: Desde la República Federal hasta 1898, Guatemala, Asociación de Amigos del País, Fundación para la Cultura y el Desarrollo, 1995, p. 697-718.

John BROWNING, «Las gazetas de Guatemala », in Historia General de Guatemala. Tomo 3: Del siglo XVIII hasta la independencia, Guatemala, Asociación de Amigos del País, Fundación para la Cultura y el Desarrollo, 1994, p. 565-578.

Gaston couté, Le gars qu'a mal tourné : poèmes et chansons, Pantin, Le Temps des cerises, 1997.

William Arlington DONOHOE, A History of British Honduras, Montréal, Provincial Publishing Co., 1946. Alejandro GómEZ, « José Cecilio del Valle : Un benthamita en Centroamérica », Anales de la academia de geografía e historia de Guatemala, t. LXXXIII, 2008, p. 83-116.

Alfredo GÓMEZ-MULLER, « Racionalidad utilitarista y emancipación: Bentham, Mill y Miranda », Carmen L. Bohórquez et Christian Ghymers (dir.), El papel de Miranda y su generación en la emancipación latinoamericana: identidad, integración regional y gobernabilidad, Caracas, Ministerio del Poder Popular para la Cultura Red de intelectuales y artistas en defensa de la humanidad, 2006, p. $265-283$.

Eric John HOBSBAWM, Nations et nationalisme depuis 1780 : programme, mythe, réalité, trad. fr. Dominique Peters, Paris, Gallimard, 2002.

Antonio José de IRISARRI, Antología, Guatemala, Academia de Geografía e Historia de Guatemala, 1999.

Hector PÉREZ BRIGNOLI (dir.), Historia general de Centroamérica, Madrid, Comunidades Europeas : Sociedad Estatal Quinto Centenario : FLACSO, 1993, vol. III De la ilustración al liberalismo (1750-1870). Joseph PÉREZ, La légende noire de l’Espagne, Paris, Fayard, 2009.

Philippe POUTIGNAT et Jocelyne STREIFF-FENART, Théories de l'ethnicité / Suivi de, « Les groupes ethniques et leurs frontières » / de Fredrik Barth., Paris, Presses Universitaires de France, 2012. Virgilio RODRÍGUEZ BETETA, Ideologías de la independencia, Ciudad Universitaria Rodrigo Facio, Costa Rica, EDUCA (Editorial Universitaria Centroamericana), 1971.

STATISTICAL INSTITUTE OF BELIZE, History of Censuses in Belize, [consulté le 19 mars 2021] ¿URL : https:// sib.org.bz/census-history-in-belize/ ${ }^{\text {. }}$.

Anne-Marie THIESSE, La création des identités nationales : Europe, $\mathrm{XVIII}^{e}-\mathrm{XX}{ }^{e}$ siècle, Paris, Éditions du Seuil, 2001. 
José TORIBIO MEDINA, La Imprenta en Guatemala (1660-1821), Guatemala, Tipografía Nacional de Guatemala, 1960.

Mario VÁZQUEZ OLIVERA, La república federal de Centro-América: territorio, nación y diplomacia, 1823-1838, Antiguo Cuscatlán, El Salvador; México, CICH-UJMD ; CIALC-UNAM, 2012.

Mario VÁZQUEZ OLIVERA, El Imperio Mexicano y el Reino de Guatemala: proyecto político y campaña militar 1821-1823, 1a. ed., México, D.F, Fondo de Cultura Económica : Universidad Nacional Autónoma de México, Centro de Investigación sobre América Latina y el Caribe, 2009.

Miriam WILLIFORD, Jeremy Bentham on Spanish America, Baton Rouge, États-Unis/Londres, Louisiana State University Press, 1980.

Josefina ZORAIDA VÁZQUEZ, « Una difícil inserción en el concierto de las naciones », Antonio Annino et François-Xavier Guerra (dir.), Inventando la nación: Iberoamérica siglo XIX, 1. ed., México, D. F, FCE - Fondo de Cultura Económica, 2003, p. 253-284.

\section{Publications périodiques}

El Indicador, Guatemala, Imprenta Nueva, 1824-1827.

El Liberal, Guatemala, Imprenta de la Unión, 1825.

Gaceta del Gobierno Supremo de Guatemala, Guatemala, Imprenta de Beteta, 1825.

Por tercera vez se reúnen D. Epifanio y D. Melitón en el cerro del Carmen, y siguen su diálogo, Guatemala, Imprenta de la Unión, 1825.

The Honduras Gazette, Belize, James Cruickshank, 1826-1827.

\section{NOTES}

1. Gaston couTÉ, Le gars qu'a mal tourné: poèmes et chansons, Pantin, Le Temps des cerises, 1997, p. 90 .

2. Benedict ANDERSON, L'Imaginaire national: réflexions sur l'origine et l'essor du nationalisme, PIERREEMMANUEL DAUZAT, Paris, Éd. La découverte, 1996.

3. Philippe POUTIGNAT et Jocelyne STREIFF-FENART, Théories de l'ethnicité / Suivi de, "Les groupes ethniques et leurs frontières " / de Fredrik Barth., Paris, Presses Universitaires de France, 2012, p. XII. 4. El Liberal, $\mathrm{n}^{\circ} 11(06 / 06 / 1825)$.

5. « Acta de independencia del antiguo reino de Guatemala », José Cecilio DEL VALLE, Obra escogida, Caracas, Biblioteca Ayacucho, 1982, p. 3.

6. Mario VÁZQUEZ OLIVERA, El Imperio Mexicano y el Reino de Guatemala: proyecto político y campaña militar 1821-1823, 1a. ed., México, D.F, Fondo de Cultura Económica: Universidad Nacional Autónoma de México, Centro de Investigación sobre América Latina y el Caribe, 2009.

7. Mario VÁzQUez olivera, La república federal de Centro-América: territorio, nación y diplomacia, 1823-1838, Antiguo Cuscatlán, El Salvador; México, CICH-UJMD ; CIALC-UNAM, 2012.

8. Hector PÉREZ BRIGNOLI (dir.), Historia general de Centroamérica, Madrid, Comunidades Europeas : Sociedad Estatal Quinto Centenario : FLACSO, 1993, vol. III De la ilustración al liberalismo (1750-1870), p. 90-118.

9. Ibid., p. 149-151.

10. William Arlington DONOHOE, A History of British Honduras, Montréal, Provincial Publishing Co., 1946, p. 27-31. 
11. STATISTICAL InSTITUTE OF BELIZE, History of Censuses in Belize [consulté le 19 mars 2021] 'URL : https://sib.org.bz/census-history-in-belize’.

12. José tORIBIO MEDINA, La Imprenta en Guatemala (1660-1821), Guatemala, Tipografía Nacional de Guatemala, 1960.

13. John BRownING, «Las gazetas de Guatemala ", Historia General de Guatemala. T. 3. Del siglo XVIII hasta la independencia, Guatemala, Asociación de Amigos del País - Fundación para la Cultura y el Desarrollo, 1994, p. 565-578.

14. Virgilio RODRíGueZ Beteta, Ideologías de la independencia, Ciudad Universitaria Rodrigo Facio, Costa Rica, EDUCA (Editorial Universitaria Centroamericana), 1971.

15. John BRoWnING, «Desarrollo del periodismo", Historia General de Guatemala. T.4. Desde la República Federal hasta 1898, Guatemala, Asociación de Amigos del País - Fundación para la Cultura y el Desarrollo, 1995, p. 697-718.

16. El Indicador, Guatemala, Imprenta Nueva, 1824-1827.

17. Pierre ALBERT, Histoire de la presse, Paris, Presses Universitaires de France, 2010.

18. Outre El Indicador, nous avons consulté l'ensemble des 32 numéros du Redactor General (1825-1826), ainsi que 24 numéros de El Liberal, 10 numéros du Semanario político-mercantil de San Salvador et 11 numéros de la Gazeta del Gobierno Supremo de Guatemala.

19. Ce discours est repris à plusieurs reprises par El Sol, par exemple, qui donne lieu à des réponses virulentes dans plusieurs des périodiques centraméricains que nous mentionnons plus bas.

20. Miriam WILLIFORD, Jeremy Bentham on Spanish America, Baton Rouge, États-Unis/Londres, Louisiana State University Press, 1980 ; Alejandro GóMEZ, « José Cecilio del Valle : Un benthamita en Centroamérica ", Anales de la academia de geografía e historia de Guatemala, tome LXXXIII, 2008, p. 83-116 ; Alfredo GóMEZ-MULLER, «Racionalidad utilitarista y emancipación: Bentham, Mill y Miranda », Carmen L. Bohórquez et Christian Ghymers (dir.), El papel de Miranda y su generación en la emancipación latinoamericana: identidad, integración regional y gobernabilidad, Caracas, Ministerio del Poder Popular para la Cultura Red de intelectuales y artistas en defensa de la humanidad, 2006, p. 265-283.

21. Josefina ZORAIDA VÁZQUEZ, « Una difícil inserción en el concierto de las naciones », in Antonio ANNINO et François-Xavier GUERRA (dir.), Inventando la nación: Iberoamérica siglo XIX, 1. ed., México, D. F, FCE - Fondo de Cultura Económica, 2003, p. 253-284.

22. "The much talked of provinces of Guatemala are in the proportion of nine tenths an uncultivated barren waste [...] full of high-sounding names of towns and cities - which exist but in name, or in clusters of huts compared with which the cabins of the poorest peasantry in Ireland are palaces ".

23. "We have given a true picture of Guatemala. We know her imbecility, her want of everything essential to make a nation, and that her fall is certain - if Great Britain does not preserve her ».

24. Appellation habituelle du Belize dans les écrits de l'époque.

25. Eric John новSваWм, Nations et nationalisme depuis 1780 : programme, mythe, réalité, trad. fr. Dominique Peters, Paris, Gallimard, 2002, p. 172.

26. asamblea nacional constituyente, Constitución de la República federal de Centro América dada por la Asamblea Nacional Constituyente en 22 de noviembre de 1824, Facismilé de l'édition de 1824., Guatemala, Imp. Marroquín Hnas., 1956, art. 12; H. PÉREZ BRIGNOLI (dir.), Historia general de Centroamérica, op. cit, p. 30.

27. "Their [the Liberals'] head was Barrundia, the philanthropic character, the all merciful and considerate man for the sufferings of his follow creatures, and whose mind was so enlarged, that he desired to make laws to govern every nation, whose system of government permitted orders in the state contrary to his judgement ».

28. «We feel, however, some pleasure in recording, that we understand those who were desirous that every justice should be done to the inhabitants of this Settlement for the injuries they 
sustained in consequence of their enactment of a law to entice our slaves from us, are the truly patriotic party, being guided in their conduct solely by the desire of benefiting their country ».

29. "Mushroom legislators who know nothing of kingdoms, of states, of men or polity ».

30. Rappelons que nous n'avons pu consulter que les numéros de El Liberal publiés en 1825.

31. ASAmblea nacional constituyente, Constitución de la República federal de Centro América dada por la Asamblea Nacional Constituyente en 22 de noviembre de 1824, op. cit.

32. EL SU-LEÑo, Por tercera vez se reúnen D. Epifanio y D. Melitón en el cerro del Carmen, y siguen su diálogo.

33. "Can England recognize, as a distinct state, a secret Colony of Spain, the only place in all America, with which she has a direct intercourse, and the decided possession of? Does Guatemala act like Colombia, or Mexico? Does she fit out vessels against Spain? Does she prohibit all communication with her or her subjects? Does she forbid the introduction of her merchandize? No; and we, therefore, repeat, that she is a secret Colony of Spain ".

34. Joseph PÉREZ, La légende noire de l'Espagne, Paris, Fayard, 2009.

35. Anne-Marie THIESSE, La création des identités nationales : Europe, $\mathrm{XVIII} \mathrm{e}^{e} \mathrm{XX} \mathrm{X}^{e}$ siècle, Paris, Éditions du Seuil, 2001, p. 11.

36. Antonio José DE IRISARRI, Antología, Guatemala, Academia de Geografía e Historia de Guatemala, 1999 , p. 78.

\section{RÉSUMÉS}

La presse périodique constitue une source de premier plan pour étudier le discours des élites politiques hispano-américaines au lendemain de l'indépendance, particulièrement dans la perspective de la construction d'une identité nationale. C'est la raison pour laquelle nous proposons d'examiner les polémiques qui sont apparues entre des journaux publiés dans la République fédérale centraméricaine d'un côté et dans le Honduras britannique voisin de l'autre en 1826 et 1827, à la suite de la parution d'une première gazette dans cet établissement colonial aujourd'hui connu sous le nom de Belize. Un échange s'engage alors entre cette gazette anglophone et la presse centraméricaine hispanophone au travers de la barrière de la langue particulièrement avec l'hebdomadaire El Indicador -, que nous analyserons sous l'angle de la volonté d'exacerber les différences de part et d'autre pour ériger une frontière de papier et affirmer de cette manière une identité culturelle et nationale.

Periodical publications constitute a primary source for studying the discourse of HispanicAmerican political elites in the aftermath of independence, particularly from the perspective of the construction of a national identity. For this reason, we suggest examining the polemics that arose between newspapers published in the Federal Republic of Central America, on the one hand, and in neighbouring British Honduras, on the other, in 1826 and 1827, following the establishment of the first gazette in the colonial settlement now known as Belize. An exchange between this anglophone gazette and the Spanish-language Central American press began across the language barrier -particularly with the weekly El Indicador- which we will analyse from the point of view of the desire to exacerbate the differences on both sides in order to erect a paper border and thus affirm a cultural and national identity. 
INDEX

Keywords : nation, independence, press, translation, Guatemala, Belize

Mots-clés : nation, indépendance, presse, traduction, Guatemala, Belize

\section{AUTEUR}

\section{RAPHAËL ROCHÉ}

Université Jean-Monnet-Saint-Étienne 